data taken by Adda, Y. [Radiation-Induced Voids in Metals. USAEC Symposium series 26, p. 40. Albany: USAEC]. For the $10^{19} \mathrm{n} / \mathrm{cm}^{2}$ samples, a gradient of 3.6 was obtained indicating that a mixture of voids and loops contributes to the small-angle scattering (dislocation loops would produce a $K^{-3}$ dependence). The size and concentration of the voids have been estimated from that portion of the Porod plot, which is linear whilst another estimate of the voids as well as the loops has been made from the low- $K$ side of the Guinier plot (Table). In the sample irradiated to $10^{19} \mathrm{n} / \mathrm{cm}^{2}$, both loops and voids are present and the concentrations in the table were derived assuming only one or the other species to be present. For the high-temperature, high-dose sample, a definite interpretation can be made in terms of voids of density $\sim 10^{14} \mathrm{~cm}^{-3}$ and diameter between $130 \AA$ and $180 \AA$.

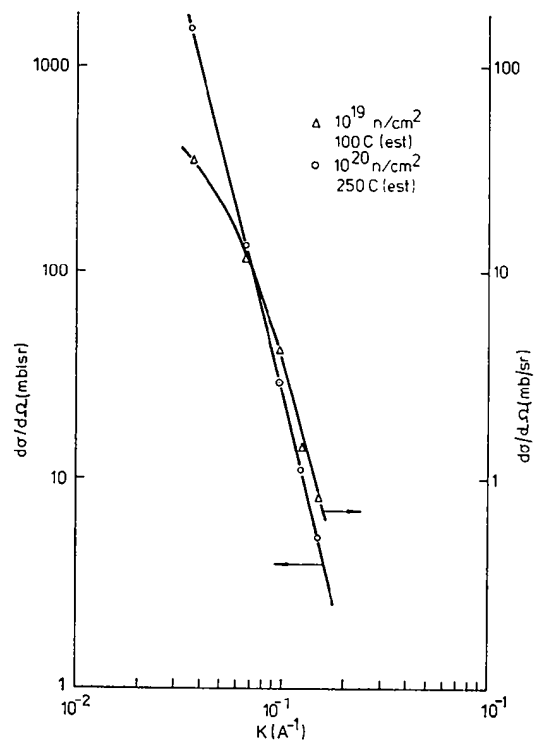

\begin{tabular}{ccccc} 
& \multicolumn{2}{c}{ Table } \\
Irradiation & Type & $\begin{array}{c}\text { Diameter } \\
(\AA)\end{array}$ & $\begin{array}{c}\text { Density } \\
\mathrm{cm}^{-3}\end{array}$ & Plot \\
$10^{19} \mathrm{n} / \mathrm{cm}^{2}$ & Loop & 85 & $4 \times 10^{16}$ & Guinier \\
$T=100 \mathrm{C}$ (est) & Void & 85 & $1 \times 10^{13}$ & Guinier \\
& & 65 & $7 \times 10^{13}$ & Porod \\
$10^{20} \mathrm{n} / \mathrm{cm}^{2}$ & Void & 130 & $6 \times 10^{14}$ & Guinier \\
$T \simeq 250 \mathrm{C}$ (est.) & & 180 & $8 \times 10^{13}$ & Porod
\end{tabular}

Figure

J. Appl. Cryst. (1975). 8, 194

\title{
The Determination of the Dislocation Structure in Copper Single Crystals from X-ray Diffraction Profiles
}

\author{
BY A. FREUND \\ Institut Max von Laue-Paul Langevin, B. P. 156 F-38042 Grenoble Cedex, France
}

(Received 29 April 1974)

\begin{abstract}
A careful analysis of X-ray diffraction profiles obtained from copper single crystals with dislocation densities between $10^{4}$ and $2 \times 10^{6}$ dislocations per $\mathrm{cm}^{2}$ has been performed. Lattice tilts and strains introduced by the as-grown dislocations have been deduced from double-crystal rocking curves recorded in a large range of Bragg angles, up to $89^{\circ}$. A new numerical deconvolution method [Mendes, M. \& Delestre, C. Submitted to Acta Cryst.] has been applied successfully to unfold the broadened diffraction patterns. The dislocation density $\varrho$ has been determined by etch-pit counting and by the application of Wilkens' theory [Wilkens, M. (1970). Phys, stat. sol. (a) 2, 359-370] to the diffraction profiles. Very good agreement has been obtained between the methods. The samples were free from low-angle grain boundaries. However, as a function of $\varrho$ the lattice tilts increased much faster than the strains, thus revealing the non-statistical distribution of the dislocations. The results indicate an arrangement of the dislocations in walls or cells as proposed by Bassim, M. N. \& Kuhlmann-Wilsdorf, D. [Cryst. Lattice Defects (1973). 4, 9-27]. The refined X-ray diffraction technique applied in this work has proved to be useful for obtaining information about the average arrangement of a great number of dislocations which is difficult to extract from topography or electron microscopy experiments. It can be used even for nearly perfect crystals.
\end{abstract}

